\title{
New Preconditioners for Nonsymmetric Saddle Point Systems with Singular $(1,1)$ Block
}

\author{
Qingbing Liu \\ Department of Mathematics, Zhejiang Wanli University, Ningbo, Zhejiang 315100, China \\ Correspondence should be addressed to Qingbing Liu; lqb_2008@hotmail.com
}

Received 27 April 2013; Accepted 28 July 2013

Academic Editors: P. Amodio, L. Hajdu, H. J. Ruskin, and K. Wang

Copyright (C) 2013 Qingbing Liu. This is an open access article distributed under the Creative Commons Attribution License, which permits unrestricted use, distribution, and reproduction in any medium, provided the original work is properly cited.

\begin{abstract}
We investigate the solution of large linear systems of saddle point type with singular $(1,1)$ block by preconditioned iterative methods and consider two parameterized block triangular preconditioners used with Krylov subspace methods which have the attractive property of improved eigenvalue clustering with increased ill-conditioning of the $(1,1)$ block of the saddle point matrix, including the choice of the parameter. Meanwhile, we analyze the spectral characteristics of two preconditioners and give the optimal parameter in practice. Numerical experiments that validate the analysis are presented.
\end{abstract}

\section{Introduction}

We study preconditioners for general nonsingular linear systems of the type

$$
\begin{gathered}
\mathscr{A} u=\left[\begin{array}{cc}
A & B^{T} \\
C & 0
\end{array}\right]\left[\begin{array}{l}
x \\
y
\end{array}\right]=\left[\begin{array}{l}
f \\
g
\end{array}\right], \\
A \in R^{n \times n}, B, C \in R^{m \times n}, n \geq m .
\end{gathered}
$$

Such systems arise in a large number of applications, for example, the (linearized) Navier-Stokes equations and other physical problems with conservation laws as well as constrained optimization problems [1-5].

As such systems are typically large and sparse, solution by iterative methods has been studied extensively $[1,5-16]$. Much attention has focused on the Navier-Stokes problem; see, for example, $[1,2,5,17,18]$. The techniques for solving systems like (1) are so numerous that it is almost impossible to give an overview. In addition to the methods developed specifically for Navier-Stokes problems, existing techniques also include splitting schemes $[2,6,12,19,20]$, constraint preconditioning [2, 20, 21], Uzawa-type algorithms [2, 12, 22], and (preconditioned) Krylov subspace methods based on (approximations to) the Schur complement $[2,16,23]$.

We start with augmentation block triangular preconditioners for the general system (1); see Section 2 for our assumptions. When $A$ is nonsingular, results for the general system have been obtained before; for example, Murphy et al. $[23,24]$ propose the block diagonal Schur complement preconditioner and the block triangular Schur complement preconditioner as follows:

$$
\mathscr{M}=\left[\begin{array}{cc}
A & 0 \\
0 & C A^{-1} B^{T}
\end{array}\right], \quad \bar{M}=\left[\begin{array}{cc}
A & 0 \\
C & C A^{-1} B^{T}
\end{array}\right] .
$$

If defined, it has been shown that the preconditioned matrices (cf. [24])

$$
\begin{gathered}
\mathscr{M}^{-1} \mathscr{A}=\left[\begin{array}{cc}
I & A^{-1} B^{T} \\
\left(C A^{-1} B^{T}\right)^{-1} C & 0
\end{array}\right], \\
\overline{\mathscr{M}}^{-1} \mathscr{A}=\left[\begin{array}{cc}
I & A^{-1} B^{T} \\
0 & -I
\end{array}\right]
\end{gathered}
$$

are diagonalizable and have only three distinct eigenvalues 1 , $(1 \pm \sqrt{5}) / 2$ and two distinct eigenvalues $1,-1$, respectively.

However, when $A$ is singular, it cannot be inverted and the Schur complement does not exist. For symmetric saddle point systems, that is, $C=B$ and $A$ is symmetric, one possible way of dealing with the systems is by augmentation, that is, by replacing $A$ with $A+B^{T} W^{-1} B$, where $W$ is an $m \times m$ symmetric positive definite weight matrix [4, 7, $9,14,17,18,25-27]$. Recently, for symmetric saddle point 
systems with $(1,1)$ block that has a high nullity, Greif and Schötzau $[25,26]$ studied the application of the following block diagonal preconditioner used with the MINRES solver for the nonsymmetric saddle point systems (1):

$$
\widehat{\mathscr{M}}=\left[\begin{array}{cc}
A+B^{T} W^{-1} B & 0 \\
0 & W
\end{array}\right] .
$$

They have shown that if the nullity of $A$ is $m$, which is the highest possible nullity, then the preconditioned matrix $\widehat{\mathscr{M}}^{-1} \mathscr{A}$ has only two distinct eigenvalues 1 and -1 . Thus, a preconditioned minimal residual Krylov subspace iterative method such as MINRES converges within two iterations.

Recently, Rees and Greif [4] presented a block triangular preconditioner as follows:

$$
\mathscr{T}=\left[\begin{array}{cc}
A+B^{T} W^{-1} B & k B^{T} \\
0 & W
\end{array}\right]
$$

and has shown that if $k=-1$, the preconditioned matrices $\mathscr{T}^{-1} \mathscr{A}$ have $\lambda=1$ with multiplicity $n-m$ and $(1 \pm \sqrt{5}) / 2$ each with multiplicity $p$. The remaining eigenvalues lie in the intervals $[(1-\sqrt{5}) / 2,0] \cup[1,(1+\sqrt{5}) / 2]$. For general nonsymmetric saddle point problems with nonsingular $(1,1)$ block, some block structured preconditioning approaches are also available [3, 4, 18, 22, 28-33].

In this paper, we propose two new block triangular preconditioners

$$
\begin{gathered}
\mathscr{A}=\left[\begin{array}{cc}
A+B^{T} W^{-1} C & k B^{T} \\
0 & -W
\end{array}\right], \\
\mathscr{A}_{k}=\left[\begin{array}{cc}
A+B^{T} W^{-1} C & k B^{T} \\
0 & (1-k) W
\end{array}\right], \quad k \neq 1,
\end{gathered}
$$

where $k$ is a scalar, and $W$ is an $m \times m$ symmetric positive definite weight matrix.

The remainder of this paper is organized as follows. In Section 2, we discuss two block triangular preconditioners for solving nonsymmetric saddle point systems, and algebraic properties are studied too. In Section 3, numerical experiments are provided to validate our analysis in Section 2. In Section 4, we draw some conclusions.

\section{Block Triangular Preconditioners}

We will adopt the general notation

$$
\mathscr{A}=\left[\begin{array}{cc}
A & B^{T} \\
C & 0
\end{array}\right]
$$

to represent the nonsymmetric saddle point matrix of (1). We assume that $A$ is symmetric and positive semidefinite with nullity $s$ and that $B$ is of size $m \times n$ and has full row rank. Note that the assumption that $\mathscr{A}$ is nonsingular implies that $\operatorname{rank}(B)=\operatorname{rank}(C)=m$, which we use in our analysis below.
We next give two lemmas in $[2,27]$.

Lemma 1 (see $[2,27])$. The nonsymmetric saddle point matrix (7)

$$
\mathscr{A}=\left[\begin{array}{cc}
A & B^{T} \\
C & 0
\end{array}\right]
$$

is nonsingular if and only if the following conditions are satisfied:

$$
\operatorname{rank}(B)=\operatorname{rank}(C)=m, \quad V_{B_{2}}^{T} A V_{C_{2}} \quad \text { nonsingular, }
$$

where $V_{B_{2}}$ and $V_{C_{2}}$ are bases of $\mathcal{N}(B)$ and $\mathcal{N}(C)$, respectively. Obviously, the nonsingularity of $V_{B_{2}}^{T} A V_{C_{2}}$ implies

$$
\begin{gathered}
\mathcal{N}(A) \cap \mathcal{N}(C)=0, \\
\mathcal{N}\left(A^{T}\right) \cap \mathscr{N}(B)=0 .
\end{gathered}
$$

Lemma 2 (see $[2,27])$. If the saddle point-type matrix $\mathscr{A}$ in (7) is nonsingular, then the rank of the matrix $A$ is at least $n-m$, and hence its nullity is at most $m$.

2.1. Block Triangular Preconditioners $\mathscr{A}$. We first consider the preconditioner

$$
\mathscr{A}=\left[\begin{array}{cc}
A+B^{T} W^{-1} C & k B^{T} \\
0 & -W
\end{array}\right] .
$$

It is easy to see that the eigenvalues of the preconditioned matrix $\mathscr{A}^{-1} \mathscr{A}$ satisfy the generalized eigenvalue problem

$$
\left[\begin{array}{cc}
A & B^{T} \\
C & 0
\end{array}\right]\left[\begin{array}{l}
u \\
v
\end{array}\right]=\lambda\left[\begin{array}{cc}
A+B^{T} W^{-1} C & k B^{T} \\
0 & -W
\end{array}\right]\left[\begin{array}{l}
u \\
v
\end{array}\right]
$$

The second block row gives $v=-(1 / \lambda) W^{-1} \mathrm{Cu}$, and substituting it into the first block equation gives

$$
\lambda(\lambda-1) A u+\left(\lambda^{2}-\lambda k+1\right) B^{T} W^{-1} C u=0 .
$$

Regardless of the choice of $k$, we see that $\lambda=1$ with algebraic multiplicity $m-m$. From the nullity of $A$ it follows that there are $s$ linearly independent null vectors of $A$. For each such null vector, we can find two $\lambda$ values satisfying $\lambda^{2}-\lambda k+1=0$. Thus, we have

$$
\lambda_{ \pm}=\frac{k \pm \sqrt{k^{2}-4}}{2}
$$

each with algebraic multiplicity $s$. The remaining $2(m-s)$ eigenvalues satisfy as following:

$$
-\frac{\lambda^{2}-\lambda}{\lambda^{2}-\lambda k+1} A u=B^{T} W^{-1} C u \text {. }
$$

Therefore, we rewrite (16) as

$$
B^{T} W^{-1} C u=\mu A x,
$$

where $-\left(\lambda^{2}-\lambda\right) /\left(\lambda^{2}-\lambda k+1\right)=\mu$. Thus, we have

$$
\lambda=\frac{(1+k \mu) \pm \sqrt{(1+k \mu)^{2}-4 \mu(1+\mu)}}{2(1+\mu)} .
$$


Since $B^{T} W^{-1} C+A$ is assumed to be nonsingular, the matrix pencil $B^{T} W^{-1} C+\varepsilon A$ is regular (cf. [27]). This expression gives an explicit formula in terms of the generalized eigenvalues of (16) and can be used to identify the intervals in which the eigenvalues lie.

To illustrate this, we consider the case $k=1$, which corresponds to setting the $(1,2)$ block of the preconditioner to be $A^{T}$. The preconditioned matrix $\mathscr{A}^{-1} \mathscr{A}$ has $\lambda=1$ with multiplicity $n-m$, and $(1 \pm \sqrt{3} i) / 2$ each with multiplicity $p$. By (18) we have

$$
\lambda=\frac{1}{2} \pm \frac{\sqrt{-3 \mu^{2}-2 \mu+1}}{2(1+\mu)} .
$$

It is worth noting that since $A$ is typically highly singular, many of the generalized eigenvalues are large, in which case the corresponding eigenvalues $\lambda_{ \pm}=(1 \pm \sqrt{3} i) / 2$ are bounded away from zero. Since the $2 s$ eigenvalues $\lambda_{ \pm}=\left(k \pm \sqrt{k^{2}-4}\right) / 2$ are unbounded as $k$ goes to $\propto$, we conclude that $k$ should be of moderate size.

2.2. Block Triangular Preconditioner $\mathscr{A}_{1}$. We next consider the preconditioner

$$
\mathscr{A}_{k}=\left[\begin{array}{cc}
A+B^{T} W^{-1} C & k B^{T} \\
0 & (1-k) W
\end{array}\right], \quad k \neq 1,
$$

where $k \neq 1$ is a scale, $W$ is an $m \times m$ symmetric positive definite weight matrix, and $A+B^{T} W^{-1} C$ is nonsingular.

The following theorem provides the spectrum results of the preconditioned matrix $\mathscr{A}_{k}^{-1} \mathscr{A}$.

Theorem 3. Let $\mathscr{A}$ be nonsingular and let its $(1,1)$ block $A$ be singular with nullitys. Then $\lambda=1$ is an eigenvalue of $\mathscr{A}_{k}^{-1} \mathscr{A}$ of geometric multiplicity $n$, and $\lambda=1 /(k-1)$ is an eigenvalue of geometric multiplicitys. The remaining $m-s$ eigenvalues satisfy the relation

$$
\lambda=-\frac{\mu}{(1-k)(\mu+1)}
$$

where $\mu$ are some $m-s$ generalized eigenvalues of the following generalized eigenvalue problem:

$$
B^{T} W^{-1} C x=\mu A x .
$$

Proof. Suppose that $\lambda$ is an eigenvalue of $\mathscr{A}_{k}^{-1} \mathscr{A}$, whose eigenvector is $\left(u^{T}, v^{T}\right)^{T}$. Then we have

$$
\mathscr{A}_{k}^{-1} \mathscr{A}\left[\begin{array}{l}
u \\
v
\end{array}\right]=\lambda\left[\begin{array}{l}
u \\
v
\end{array}\right] \text {. }
$$

Furthermore, it satisfies the generalized eigenvalue problem

$$
\left[\begin{array}{cc}
A & B^{T} \\
C & 0
\end{array}\right]\left[\begin{array}{l}
u \\
v
\end{array}\right]=\lambda\left[\begin{array}{cc}
A+B^{T} W^{-1} C & k B^{T} \\
0 & (1-k) W
\end{array}\right]\left[\begin{array}{l}
u \\
v
\end{array}\right]
$$

or

$$
\begin{gathered}
A u+B^{T} v=\lambda\left(A+B^{T} W^{-1} C\right) u+\lambda k B^{T} v \\
C u=\lambda(1-k) W v .
\end{gathered}
$$

As $\mathscr{A}_{k}$ is nonsingular, $\lambda \neq 0$. The second equality gives that $v=$ $(1 / \lambda(1-k)) W^{-1} C u$, and substituting it into the first equality gives

$$
(\lambda-1)\left[\lambda(1-k) A u-(\lambda k-\lambda-1) B^{T} W^{-1} C u\right]=0 .
$$

It is straightforward to see that any vector $u \in R^{n}$ satisfies (26) with $\lambda=1$, and thus $\lambda=1$ is an eigenvalue of $\mathscr{A}_{k}^{-1} \mathscr{A}$. We claim that the eigenvalue $\lambda=1$ has geometric multiplicity $n$.

If $\lambda \neq 1$, then from (26) we have

$$
\lambda(1-k) A u+(\lambda k-\lambda-1) B^{T} W^{-1} C u=0 .
$$

If $u \in \mathcal{N}(A)$, then from (27) we have

$$
(\lambda k-\lambda-1) B^{T} W^{-1} C u=0 .
$$

From Lemma 1 and (28), $A$ is singular with nullity $s$, we know that $\lambda=1 /(k-1)$ is an eigenvalue of $\mathscr{A}_{k}^{-1} \mathscr{A}$ of geometric multiplicity $s$.

We have determined $n+s$ eigenvalues. Now we consider the remaining $m-s$ eigenvalues of $\mathscr{A}_{k}^{-1} \mathscr{A}$.

Suppose that $\lambda \neq 1,1 /(k-1)$, then from (27) we have

$$
B^{T} W^{-1} C u=\mu A u,
$$

where $\mu=\lambda(1-k) /(\lambda k-\lambda-1)$, which implies that $\lambda=-\mu /(1-$ $k)(\mu+1)$. Since $B^{T} W^{-1} C+A$ is assumed to be nonsingular, the matrix pencil $B^{T} W^{-1} C+\varepsilon A$ is regular (cf. [27]). Thus, the generalized eigenvalue problem (29) is well posed.

If $k=0$, then we have the following corollary.

Corollary 4. Let $\mathscr{A}$ be nonsingular and let its $(1,1)$ block $A$ be singular with nullity s. Then $\lambda=1$ is an eigenvalue of $\mathscr{A}_{0}^{-1} \mathscr{A}$ of geometric multiplicity $n$, and $\lambda=-1$ is an eigenvalue of geometric multiplicitys. The remaining $m-s$ eigenvalues satisfy the relation

$$
\lambda=-\frac{\mu}{\mu+1}
$$

where $\mu$ are some $m$ - s generalized eigenvalues of the following generalized eigenvalue problem:

$$
B^{T} W^{-1} C x=\mu A x
$$

From Theorem 3 and Corollary 4 we know that the higher the nullity of $A$ is, the stronger the clustered eigenvalues of $\mathscr{A}_{0}^{-1} \mathscr{A}$ are. When the nullity of $A$ is $m$, its at most value (cf. Lemma 2), we have the following result.

Corollary 5. Let $\mathscr{A}$ be nonsingular and let its $(1,1)$ block $A$ be singular with nullity $m$. Then the preconditioned matrix $\mathscr{A}_{0}^{-1} \mathscr{A}$ is diagonalizable and has precisely two eigenvalues $\lambda=1$ of geometric multiplicity $n$ and $\lambda=-1$ of geometric multiplicity $m$. 
If $k=2$, we have the following corollary.

Corollary 6. Let $\mathscr{A}$ be nonsingular and let its $(1,1)$ block $A$ be singular with nullity s. Then $\lambda=1$ is an eigenvalue of $\mathscr{A}_{2}^{-1} \mathscr{A}$ of geometric multiplicity $n+s$. The remaining $m-s$ eigenvalues satisfy the relation

$$
\lambda=\frac{\mu}{\mu+1},
$$

where $\mu$ are some $m-$ s generalized eigenvalues of the following generalized eigenvalue problem:

$$
B^{T} W^{-1} C x=\mu A x .
$$

From Theorem 3 and Corollary 6 , if the nullity of $A$ is $m$, we have the following corollary.

Corollary 7. Let $\mathscr{A}$ be nonsingular and let its $(1,1)$ block $A$ be singular with nullitym. Then the preconditioned matrix $\mathscr{A}_{2}^{-1} \mathscr{A}$ has only precisely one eigenvalue $\lambda=1$ of geometric multiplicity $n+m$.

Remark 8. From Corollaries 5 and 7, if the nullity of $A$ is $m$, then it is readily seen that the preconditioned matrix $\mathscr{A}_{0}^{-1} \mathscr{A}$ has precisely two distinct eigenvalues and that the preconditioned matrix $\mathscr{A}_{2}^{-1} \mathscr{A}$ has only precisely one eigenvalue. Thus, we know that any preconditioned Krylov subspace method such as GMRES terminates in at most two steps if roundoff errors are ignored.

\section{Numerical Experiments}

In this section we present numerical experiments to illustrate the performance of the two preconditioners when they are implemented exactly or approximately.

For our experiments, as in $[19,27]$ we construct the saddle point-type matrix $\widetilde{\mathscr{A}}$ from reforming a matrix $\mathscr{A}$ of the following form:

$$
\mathscr{A}=\left[\begin{array}{cc}
A & B^{T} \\
B & 0
\end{array}\right]
$$

where the matrix $\mathscr{A}$ arises from the discretization of the Stokes problem as follows:

$$
\begin{gathered}
-\mu \Delta \mathbf{u}+\nabla w=\widetilde{f}, \quad \text { in } \Omega, \\
\nabla \cdot \mathbf{u}=\widetilde{g}, \quad \text { in } \Omega, \\
\mathbf{u}=0, \quad \text { on } \partial \Omega, \\
\int_{\Omega} w(x) d x=0,
\end{gathered}
$$

where $\Omega=(0,1) \times(0,1) \subset R^{2}, \partial \Omega$ is the boundary of $\Omega, \Delta$ is the componentwise Laplace operator, $u$ is a vector-valued function representing the velocity, and $w$ is a scalar function representing the pressure ( $\mathrm{cf}[19])$. Then the matrices $B$ and $B^{T}$ are replaced by a random matrix $\widehat{C}$ with the same sparsity as $B$ and a random matrix $\widehat{B}^{T}$ with the same sparsity as $B^{T}$, respectively. Furthermore, $\widehat{C}(1: m, 1: m)$ and $\widehat{B}(1: m, 1: m)$ are, respectively, replaced by $C_{1}=\widehat{C}(1: m, 1: m)-(3 / 2) I_{m}$ and $B_{1}=\widehat{B}(1: m, 1: m)-(3 / 2) I_{m}$, such that $C_{1}$ and $B_{1}$ are nonsingular. Denote $C_{2}=\widehat{C}(1: m, m+1: n)$ and $B_{2}=\widehat{B}(1$ : $m, m+1: n)$; then we have $C=\left[C_{1}, C_{2}\right]$ and $B=\left[B_{1}, B_{2}\right]$ with $B_{1}, C_{1} \in R^{m, m}$ and $B_{2}, C_{2} \in R^{m, n-m}$. Obviously, the resulting saddle point-type matrix

$$
\widetilde{A}=\left[\begin{array}{cc}
A & B^{T} \\
C & 0
\end{array}\right]
$$

satisfies

$$
\operatorname{rank}(C)=\operatorname{rank}(B)=m .
$$

From the matrix $\widetilde{\mathscr{A}}$ in (36) we construct the following four saddle point-type matrices:

$$
\widetilde{\mathscr{A}}_{i}=\left[\begin{array}{cc}
A_{i} & B^{T} \\
C & 0
\end{array}\right], \quad i=1, \ldots, 4,
$$

where $A_{i}$ is constructed from $A$ by making its $i \times(m / 4)$ rows and columns with zero entries. Note that $A_{i}$ is semipositive real and its nullity is $i \times(\mathrm{m} / 4)$.

In the first numerical example, the matrix $W$ in the augmentation block preconditioners is taken as $W=(1 / \gamma) I_{m}$, whereas the positive parameter $\gamma$ is taken as (cf. [17])

$$
\gamma=\frac{\left\|A_{i}\right\|_{1}}{\|B\|_{1}\|C\|_{1}} .
$$

Theorem 3 and Corollaries 4-7 guarantee increased spectral clustering of the preconditioned matrix $\mathscr{A}_{k}^{-1} \mathscr{A}$ when $k=2$. As expected by the previous theorems and corollaries, Figures 1 and 2 plot, respectively, the two preconditioned matrices, each figure includes four subfigures that plot, respectively, the eigenvalues of four preconditioned matrices resulting from four matrices $\widetilde{\mathscr{A}}_{i}, i=1, \ldots, 4$, operated upon by exactly the same preconditioner.

From these figures we can clearly see that the higher the nullity of the $(1,1)$ block, the more strongly the eigenvalues of the preconditioned matrices are clustered. Furthermore, we note that Figures 1 and 2 show that $\lambda=1$ has very high multiplicity when the nullity of the $(1,1)$ block $A_{i}$ is $m$.

Next, we study the iteration numbers and iteration time for $\widetilde{\mathscr{A}}_{3}$ and $\widetilde{\mathscr{A}}_{4}$. Table 1 shows results for applying GMRES of block triangular preconditioners $\mathscr{A}$. From Table 1, we can see that the preconditioned GMRES of $\mathscr{A}^{-1} \mathscr{A}$ is more efficient when $k=2$, and iteration numbers are slightly changed by the change of parameter $k$ when the nullity of $A$ is $m$ (see Tables 1 and 2).

We next will use preconditioned GMRES to solve, respectively, the following four saddle point-type systems:

$$
\widetilde{\mathscr{A}}_{i} x=f_{i}, \quad i=3,4,
$$

where the right-hand side $f_{i}$ is taken such that the exact solution $x$ is equal to $(1,1, \ldots, 1)^{T}$. The stopping criterion is 


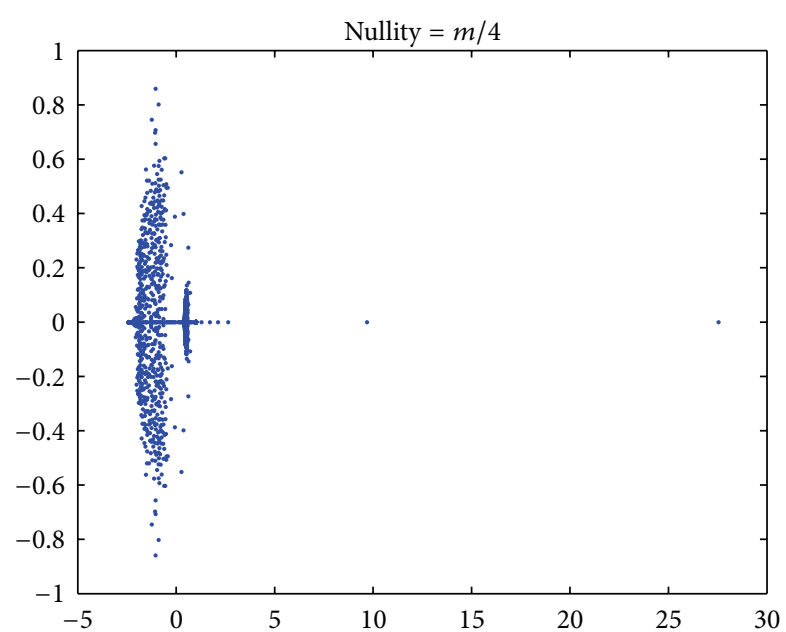

(a)

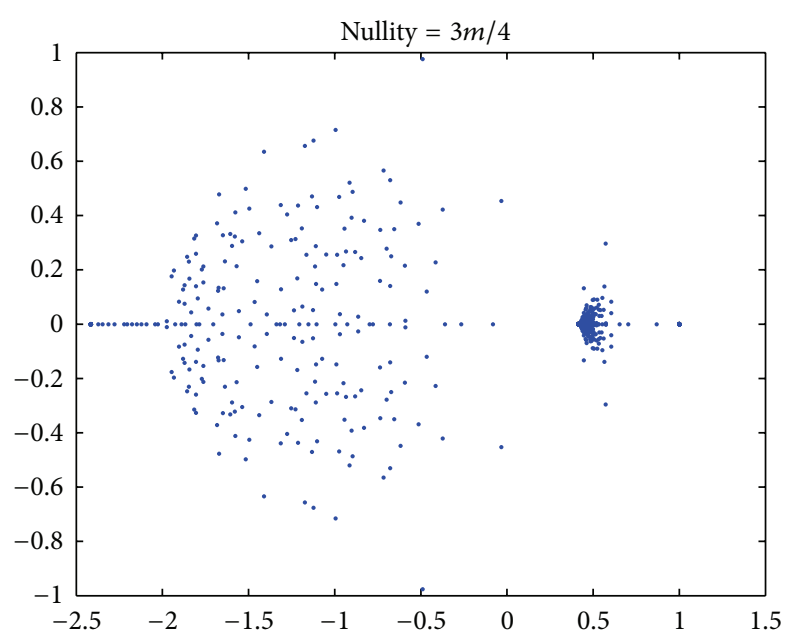

(c)

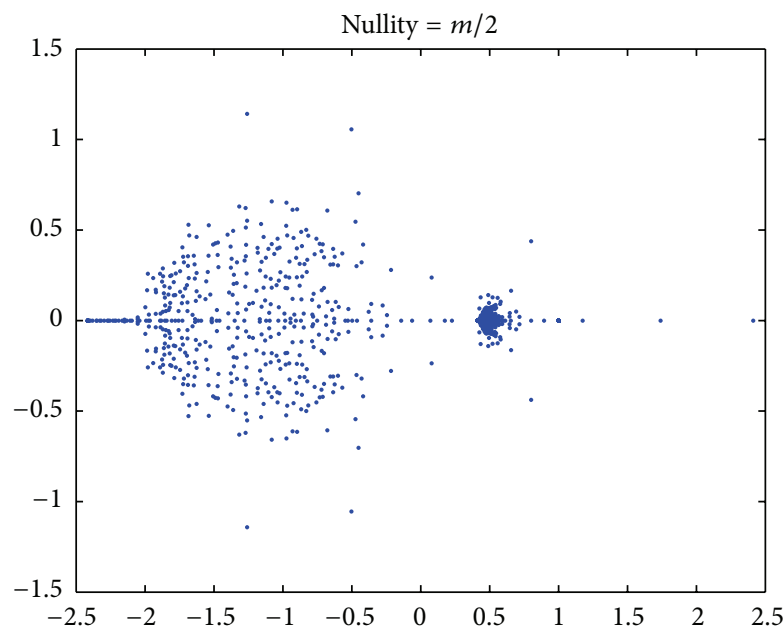

(b)

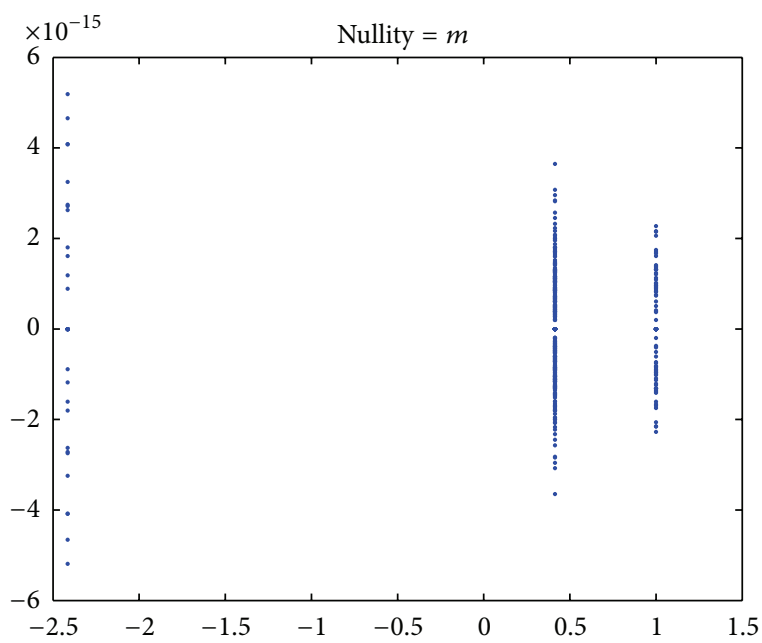

(d)

FIGURE 1: The eigenvalue distribution of preconditioned systems $\mathscr{T}^{-1} \mathscr{A}$ with $k=2$ and $m+n=2700$.

TABLE 1: Iteration time and iteration numbers of GMRES of block triangular preconditioner $\mathscr{A}$ for different $k$ with $m+n=1200$.

\begin{tabular}{lcccccc}
\hline Nullity & $k$ & 0.5 & 1 & 1.5 & 2 & 2.5 \\
\hline \multirow{2}{*}{$3 m / 4$} & Time & 102.9954 & 126.6284 & 63.4525 & 36.4118 & 54.9560 \\
& Iter & 16 & 18 & 9 & 5 & 5 \\
\hline \multirow{2}{*}{$m$} & Time & 2.8677 & 2.8821 & 3.0962 & 2.3289 & 3.0844 \\
& Iter & 1 & 1 & 1 & 1 \\
\hline
\end{tabular}

TABLE 2: Iteration time and iteration numbers of GMRES of preconditioned matrices $\mathscr{T}^{-1} \mathscr{A}$ and $\mathscr{A}^{-1} \mathscr{A}$ for different $k=2$ with nullity $=3 m / 4$.

\begin{tabular}{ccccccc}
\hline & $m+n$ & 300 & 675 & 1200 & 2028 & 2700 \\
\hline \multirow{2}{*}{$\mathscr{T}$} & Time & 0.3066 & 14.3040 & 3449.2035 & $>3000$ & $>5000$ \\
& Iter & 10 & 100 & $>1000$ & 25000 & $>1000$ \\
\hline \multirow{2}{*}{$\mathscr{A}^{-1} \mathscr{A}$} & Time & 0.0667 & 0.4282 & 2.0694 & 1519.8529 \\
& Iter & 2 & 3 & 3 & 7 & 17 \\
\hline
\end{tabular}




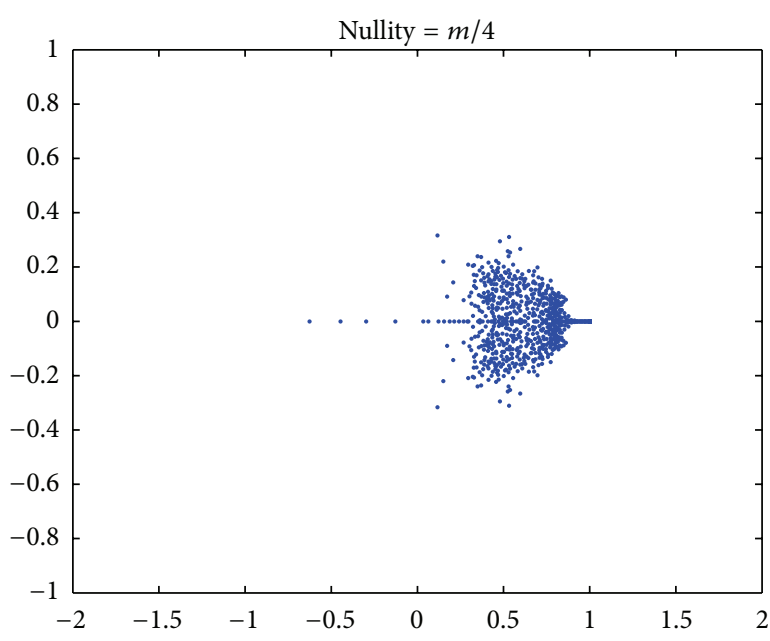

(a)

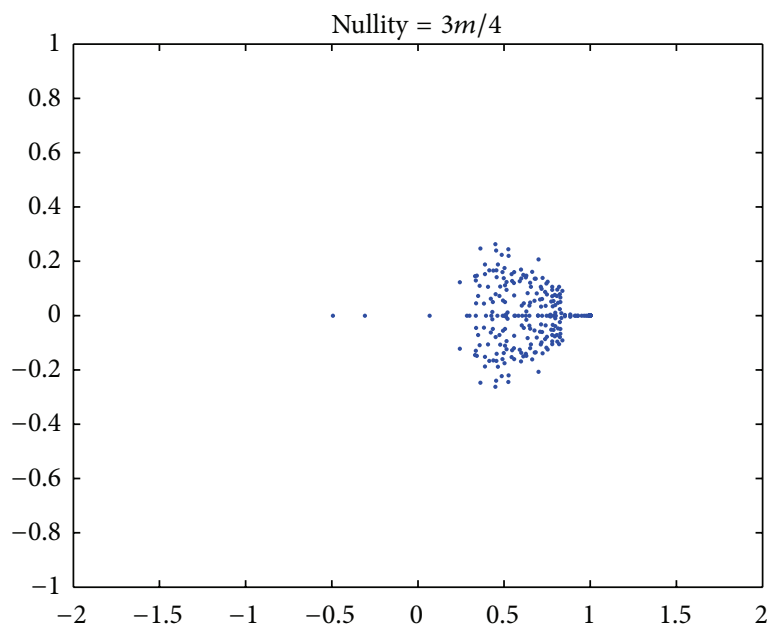

(c)

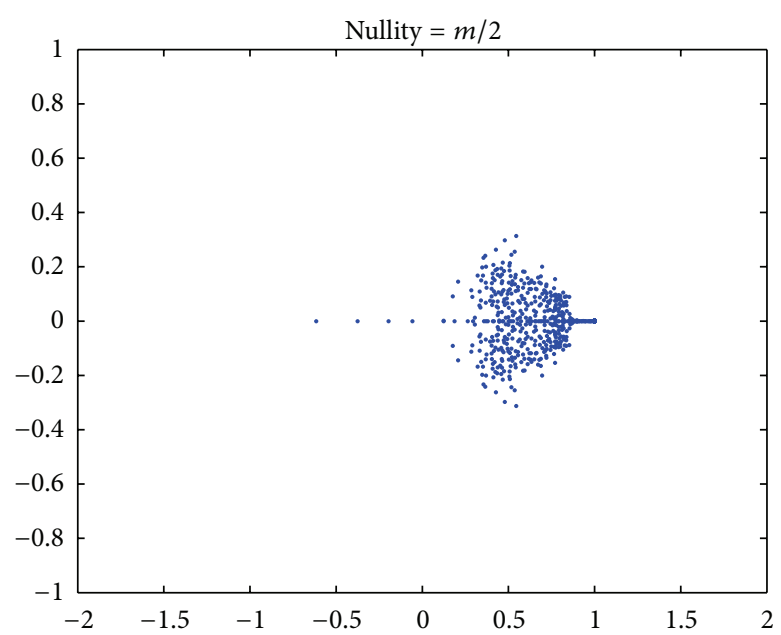

(b)

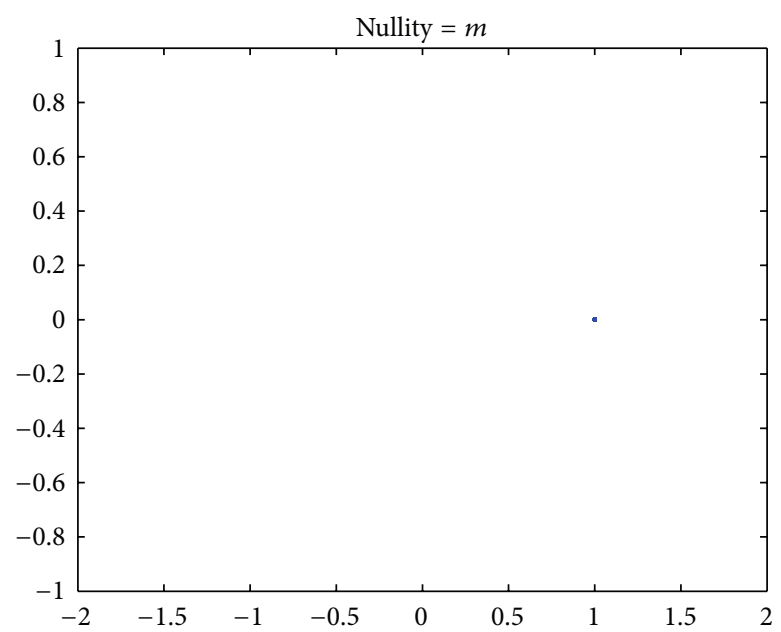

(d)

FIGURE 2: The eigenvalue distribution of preconditioned systems $\mathscr{A}^{-1} \mathscr{A}$ with $k=2$ and $m+n=2700$.

TABLE 3: Iteration time and iteration numbers of GMRES of preconditioned matrices $\mathscr{T}^{-1} \mathscr{A}$ and $\mathscr{A}^{-1} \mathscr{A}$ for different $k=2$ with nullity $=m$.

\begin{tabular}{ccccccc}
\hline & $m+n$ & 300 & 1200 & 2028 & 2700 & 3675 \\
\hline \multirow{2}{*}{$\mathscr{T}^{-1} \mathscr{A}$} & Time & 0.3856 & 2.7447 & 11.7693 & 31.8844 & 15.5760 \\
& Iter & 1 & 1 & 1 & 1 & 1 \\
\hline \multirow{2}{*}{$\mathscr{A}^{-1} \mathscr{A}$} & Time & 0.0752 & 2.2487 & 9.3957 & 20.9046 & 64.8728 \\
& Iter & 1 & 1 & 1 & 1 \\
\hline
\end{tabular}

$\left\|r^{(k)}\right\| /\left\|f_{i}\right\| \leq 10^{-6}$ and $r^{(k)}$ is the residual vector after $k$ th iteration. In this section, let $k=2$; we give some comparison results of the iteration numbers and iteration time for five preconditioners (see Tables 2 and 3). From Table 2, when the nullity of $A$ is $3 \mathrm{~m} / 4$, we see that the preconditioned GMRES with the preconditioner $\mathscr{A}$ is more efficient than that of the preconditioner $\mathscr{T}$, but from Table 3, when the nullity of $A$ is $m$, we see that the preconditioned GMRES with the preconditioner $\mathscr{A}$ is essentially similar to the preconditioner $\mathscr{T}$ in iteration time and iteration numbers.
All the numerical experiments were performed with MATLAB 7.0. The machine we have used is a PC-AMD, CPU T7400 2.2 GHz process.

\section{Conclusions}

In this paper, we have analyzed the spectral properties as well as the computational performance of two types of block triangular augmentation preconditioners for saddle pointtype matrices with a highly singular $(1,1)$ block. Complete 
theoretical analysis shows that all eigenvalues of the preconditioned matrices are strongly clustered. A good parameter choice may substantially reduce the iteration numbers and iteration time. In particulare, we have shown that in cases where the $(1,1)$ block has high nullity, convergence for each of the two preconditioned GMRES iterative methods is guaranteed to be almost immediate. Numerical experiments are also reported for illustrating the efficiency of the presented preconditioners. From the views of theories and applications, the presented preconditioners are better than the previous results.

\section{Acknowledgments}

The author wishes to thank the editor Professor Kamal Mostafa and the anonymous referees for their helpful suggestions which improved this paper. This research was supported by the National Natural Science Foundation of China (no. 11071079), the Ningbo Natural Science Foundation (2012A610037) and the Fundamental Research Funds for the Central Universities.

\section{References}

[1] O. Axelsson, "Preconditioning of indefinite problems by regularization," SIAM Journal on Numerical Analysis, vol. 16, pp. 5869, 1979.

[2] M. Benzi, G. H. Golubt, and J. Liesen, "Numerical solution of saddle point problems," Acta Numerica, vol. 14, pp. 1-137, 2005.

[3] P. E. Gill, W. Murray, D. B. Ponceleon, and M. A. Saunders, "Preconditioners for indefinite systems arising in optimization," SIAM Journal on Matrix Analysis and Applications, vol. 13, pp. 292-311, 1992.

[4] T. Rees and C. Greif, "A preconditioner for linear systems arising from interior point optimization methods," SIAM Journal on Scientific Computing, vol. 29, no. 5, pp. 1992-2007, 2007.

[5] A. Wathen and D. Silvester, "Fast iterative solution of stabilized stokes systems part I: using simple diagonal preconditioners," SIAM Journal on Numerical Analysis, vol. 30, no. 3, pp. 630-649, 1993.

[6] Z. Bai, G. H. Golub, and M. K. Ng, "Hermitian and skewHermitian splitting methods for non-Hermitian positive definite linear systems," SIAM Journal on Matrix Analysis and Applications, vol. 24, no. 3, pp. 603-626, 2003.

[7] G. H. Golub and C. Greif, "On solving block-structured indefinite linear systems," SIAM Journal on Scientific Computing, vol. 24, no. 6, pp. 2076-2092, 2003.

[8] B. J. Bunch and B. N. Parlett, "Direct methods for solving symmetric indefinite systems of linear equations," SIAM Journal on Numerical Analysis, vol. 8, no. 4, pp. 639-655, 1971.

[9] Z. Chen and J. Zou, "An augmented lagrangian method for identifying discontinuous parameters in elliptic systems," SIAM Journal on Control and Optimization, vol. 37, no. 3, pp. 892-910, 1999.

[10] P. Ciarlet Jr., J. Huang, and J. Zou, "Some observations on generalized saddle-point problems," SIAM Journal on Matrix Analysis and Applications, vol. 25, no. 1, pp. 224-236, 2004.

[11] I. S. Duff and J. K. Reid, "Exploiting zeros on the diagonal in the direct solution of indefinite sparse symmetric linear systems,"
ACM Transactions on Mathematical Software, vol. 22, no. 2, pp. 227-257, 1996.

[12] G. H. Golub, X. Wu, and J. Yuan, "SOR-like methods for augmented systems," BIT Numerical Mathematics, vol. 41, no. 1, pp. 71-85, 2001.

[13] W. Hackbusch, Iterative Solution of Large Sparse Systems of Equations, Springer, Berlin, Germany, 1994.

[14] Q. Hu, Z. Shi, and D. Yu, "Efficient solvers for saddle-point problems arising from domain decompositions with lagrange multipliers," SIAM Journal on Numerical Analysis, vol. 42, no. 3, pp. 905-933, 2004.

[15] C. C. Paige and M. A. Saunders, "Solution of sparse indefinite systems of linear equations," SIAM Journal on Numerical Analysis, vol. 12, no. 4, pp. 617-629, 1975.

[16] Y. Saad, Iterative Methods for Sparse Linear Systems, SIAM, Philadelphia, Pa, USA, 2nd edition, 2003.

[17] M. Benzi and J. Liu, "Block preconditioning for saddle point systems with indefinite $(1,1)$ block," International Journal of Computer Mathematics, vol. 84, no. 8, pp. 1117-1129, 2007.

[18] G. Cheng, T. Huang, and S. Shen, "Block triangular preconditioners for the discretized time-harmonic Maxwell equations in mixed form," Computer Physics Communications, vol. 180, no. 2, pp. 192-196, 2009.

[19] Z. Bai, G. H. Golub, and J. Pan, "Preconditioned Hermitian and skew-Hermitian splitting methods for non-Hermitian positive semidefinite linear systems," Numerische Mathematik, vol. 98, no. 1, pp. 1-32, 2004.

[20] Z. Bal, M. K. Ng, and Z. Wang, "Constraint preconditioners for symmetric indefinite matrices," SIAM Journal on Matrix Analysis and Applications, vol. 31, no. 2, pp. 410-433, 2009.

[21] C. Keller, N. I. M. Gould, and A. J. Wathen, "Constraint preconditioning for indefinite linear systems," SIAM Journal on Matrix Analysis and Applications, vol. 21, no. 4, pp. 1300-1317, 2000.

[22] A. Frangioni and C. Gentile, "New preconditioners for KKT systems of network flow problems," SIAM Journal on Optimization, vol. 14, no. 3, pp. 894-913, 2004.

[23] I. C. F. Ipsen, "A note on preconditioning nonsymmetric matrices," SIAM Journal on Scientific Computing, vol. 23, no. 3, pp. 1050-1051, 2002.

[24] M. F. Murphy, G. H. Golub, and A. J. Wathen, "Note on preconditioning for indefinite linear systems," SIAM Journal on Scientific Computing, vol. 21, no. 6, pp. 1969-1972, 2000.

[25] C. Greif and D. Schötzau, "Preconditioners for saddle point linear systems with highly singular $(1,1)$ blocks," Electronic Transactions on Numerical Analysis, vol. 22, pp. 114-121, 2006.

[26] C. Greif and D. Schötzau, "Preconditioners for the discretized time-harmonic Maxwell equations in mixed form," Numerical Linear Algebra with Applications, vol. 14, no. 4, pp. 281-297, 2007.

[27] Z. Cao, "Augmentation block preconditioners for saddle pointtype matrices with singular $(1,1)$ blocks," Numerical Linear Algebra with Applications, vol. 15, no. 6, pp. 515-533, 2008.

[28] H. C. Elman, D. J. Silvester, and A. J. Wathen, "Performance and analysis of saddle point preconditioners for the discrete steadystate Navier-Stokes equations," Numerische Mathematik, vol. 90, no. 4, pp. 665-688, 2002.

[29] R. W. Freund, "Preconditioning of symmetric, but highly indefinite linear systems," in Proceedings of the 15th IMACS World Congress on Scientific Computation, Modeling and Applied Mathematics, pp. 551-556, 1997. 
[30] A. Klawonn and G. Starke, "Block triangular preconditioners for nonsymmetric saddle point problems: field-of-values analysis," Numerische Mathematik, vol. 81, no. 4, pp. 577-594, 1999.

[31] V. Simoncini, "Block triangular preconditioners for symmetric saddle-point problems," Applied Numerical Mathematics, vol. 49, no. 1, pp. 63-80, 2004.

[32] E. De Sturler and J. Liesen, "Block-diagonal and constraint preconditioners for nonsymmetric indefinite linear systems. Part I: theory," SIAM Journal on Scientific Computing, vol. 26, no. 5, pp. 1598-1619, 2005.

[33] K. Toh, K. Phoon, and S. Chan, "Block preconditioners for symmetric indefinite linear systems," International Journal for Numerical Methods in Engineering, vol. 60, no. 8, pp. 1361-1381, 2004. 


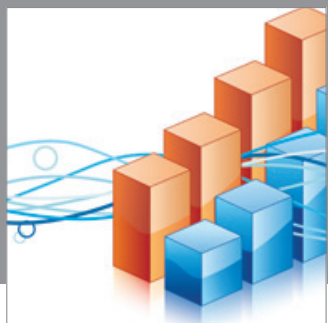

Advances in

Operations Research

mansans

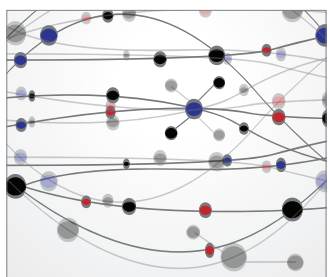

The Scientific World Journal
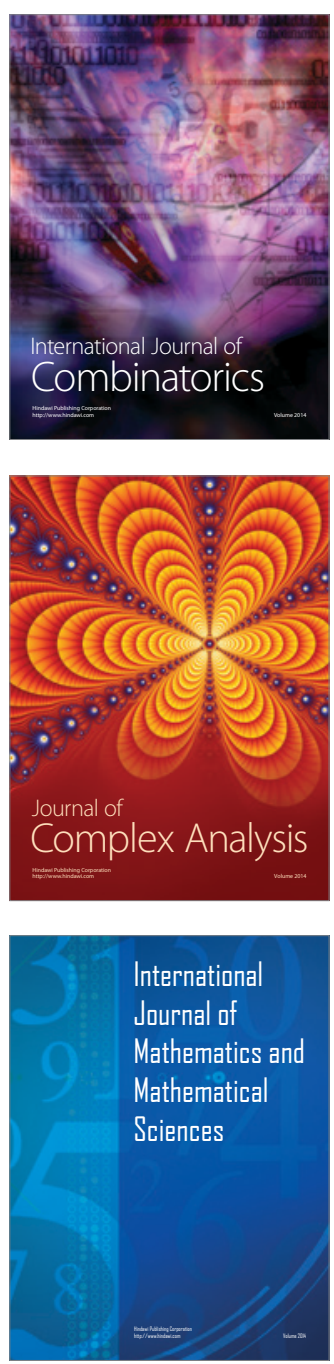
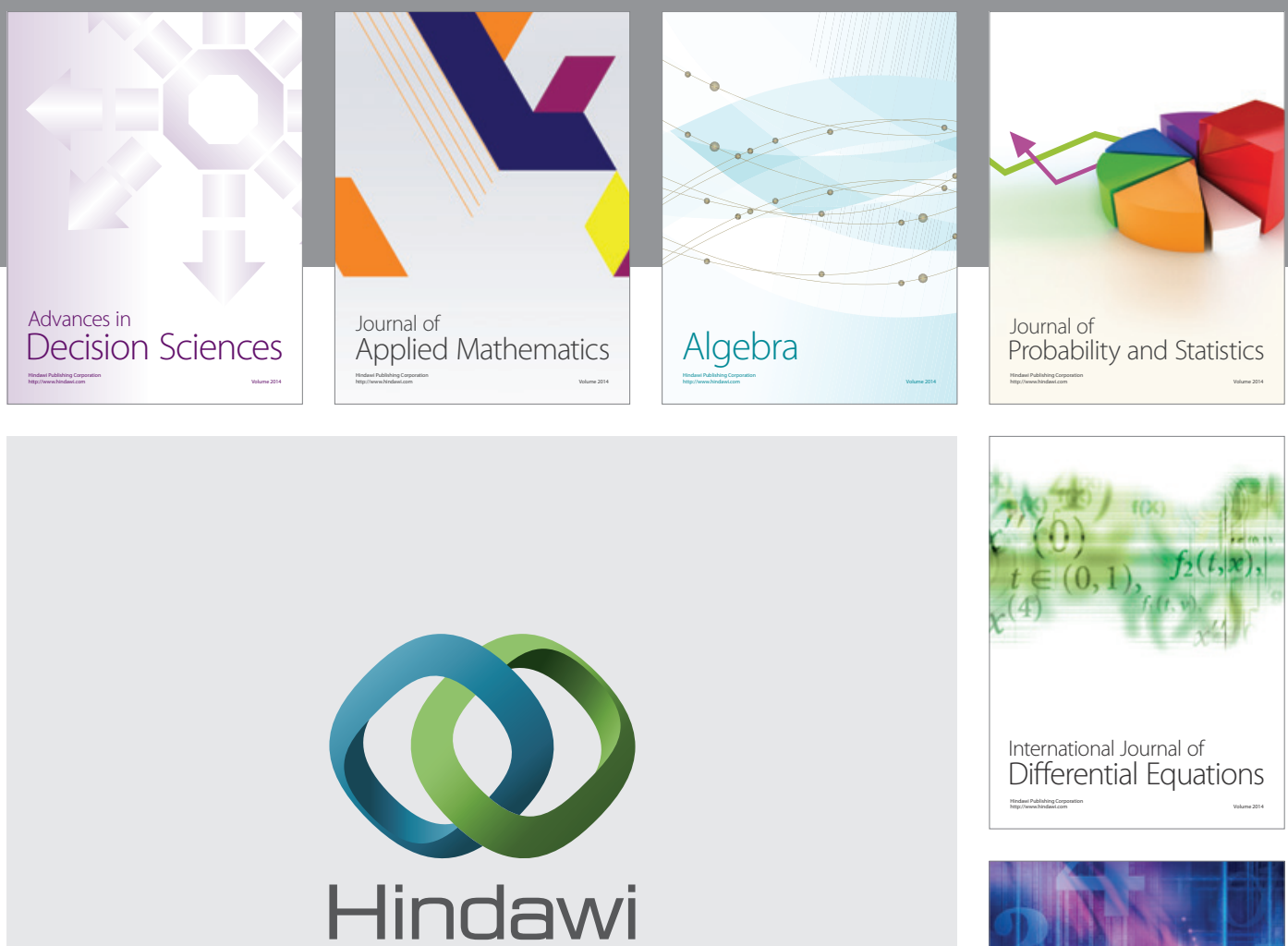

Submit your manuscripts at http://www.hindawi.com
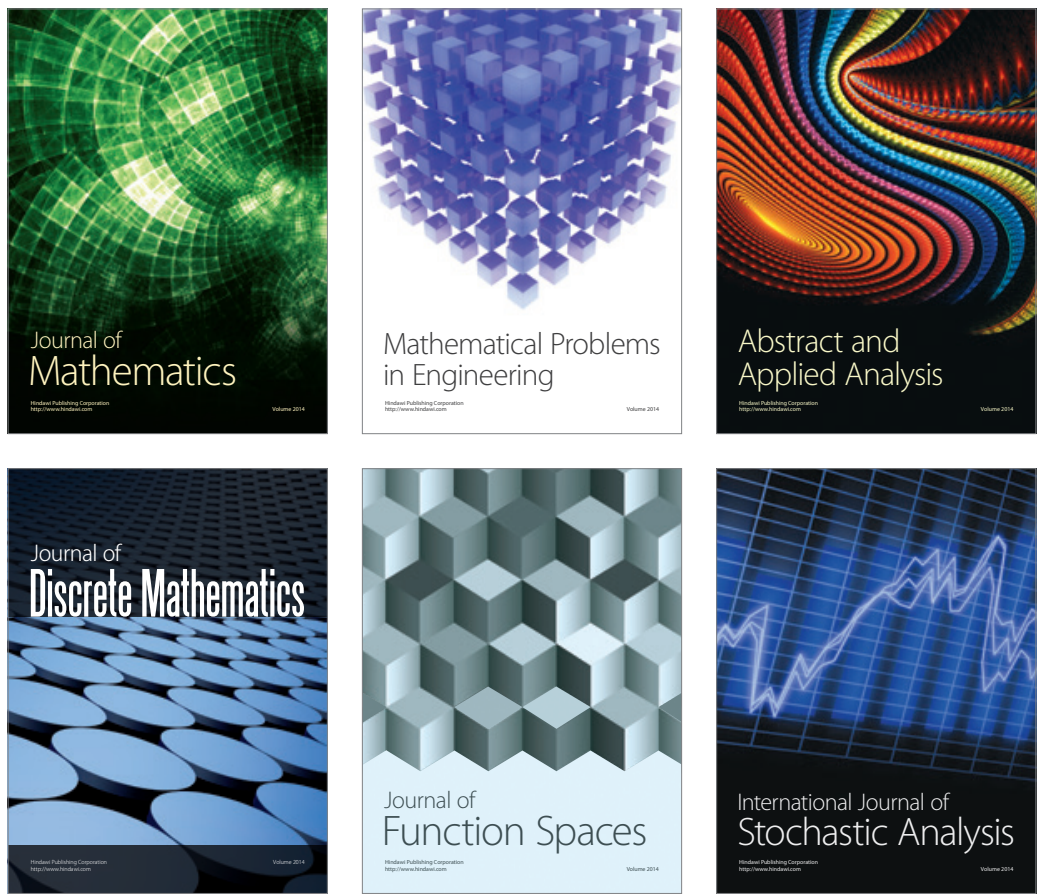

Journal of

Function Spaces

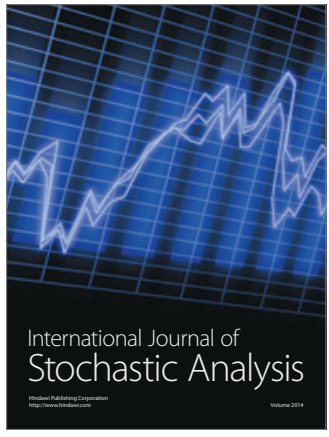

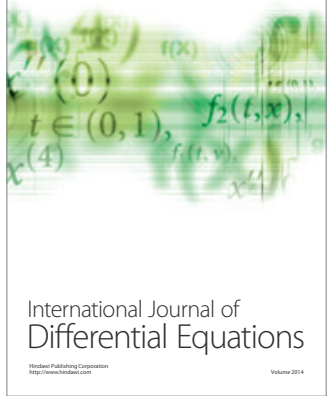
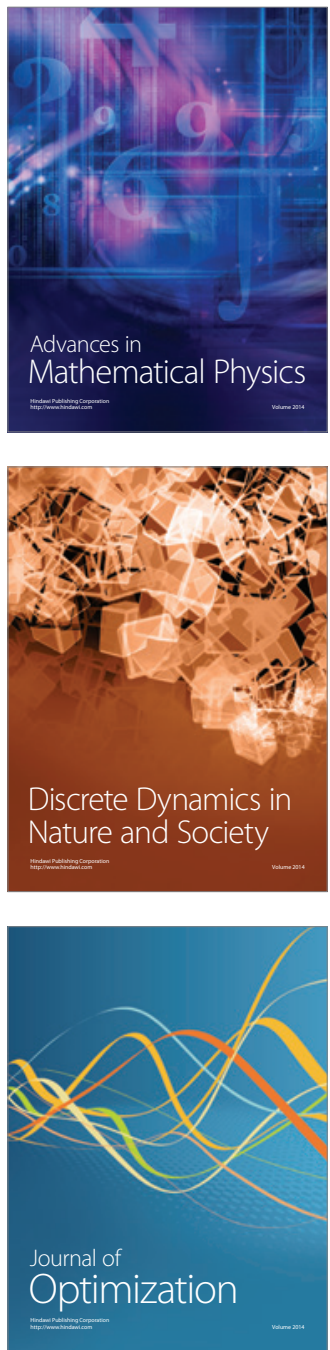